\title{
Intranasal inhibitor blocks Omicron and other variants of SARS-CoV-2
}

Anna R. Mäkelä ${ }^{1}$, Hasan Uğurlu ${ }^{1}$, Liina Hannula², Petja Salminen ${ }^{1}$, Ravi Kant ${ }^{1,3}$, Riku Fagerlund ${ }^{1}$, Anu Haveri ${ }^{4}$, Tomas Strandin ${ }^{1}$, Lauri Kareinen ${ }^{1,3}$, Jussi Hepojoki ${ }^{1}$, Lev Levanov ${ }^{1}$, Arja Pasternack $^{5}$, Rauno A. Naves 5 , Olli Ritvos ${ }^{5}$, Pamela Österlund ${ }^{4}$, Tarja Sironen ${ }^{1,3}$, Olli Vapalahti $^{1,3,7}$, Anja Kipar ${ }^{3,6}$, Juha T. Huiskonen ${ }^{2}$, Ilona Rissanen ${ }^{2}$ and Kalle Saksela ${ }^{1,7}$

${ }^{1}$ Department of Virology, University of Helsinki, Helsinki, Finland

${ }^{2}$ Institute of Biotechnology, Helsinki Institute of Life Science HiLIFE, University of Helsinki, Helsinki, Finland.

${ }^{3}$ Department of Basic Veterinary Sciences, University of Helsinki, Helsinki, Finland

${ }^{4}$ Finnish Institute for Health and Welfare, Helsinki, Finland.

${ }^{5}$ Department of Physiology, University of Helsinki, Helsinki, Finland

${ }^{6}$ Laboratory for Animal Model Pathology, Institute of Veterinary Pathology, Vetsuisse Faculty, University of Zurich, Zurich, Switzerland

${ }^{7}$ HUS Diagnostic Centre, HUSLAB, Clinical Microbiology, Helsinki University Hospital, Helsinki, Finland.

*Correspondence: kalle.saksela@helsinki.fi 


\section{SUMMARY}

The emergence of the SARS-CoV-2 Omicron variant capable of escaping neutralizing antibodies emphasizes the need for prophylactic strategies to complement vaccination in fighting the COVID19 pandemic. Nasal epithelium is rich in the ACE2 receptor and important for SARS-CoV-2 transmission by supporting early viral replication before seeding to the lung ${ }^{1}$. Intranasal administration of SARS-CoV-2 neutralizing antibodies or antibody fragments has shown encouraging potential as a protective measure in animal models ${ }^{2-5}$. However, there remains a need for SARS-CoV-2 blocking agents that are more economical to produce in large scale, while less vulnerable to mutational variation in the neutralization epitopes of the viral Spike glycoprotein. Here we describe TriSb92, a highly manufacturable trimeric human nephrocystin SH3 domainderived antibody mimetic targeted against a conserved region in the receptor-binding domain of the Spike. TriSb92 potently neutralizes SARS-CoV-2 and its variants of concern, including Delta and Omicron. Intranasal administration of a modest dose of TriSb92 (5 or 50 micrograms) as early as eight hours before the challenge with SARS-CoV-2 B.1.351 efficiently protected mice from infection. The target epitope of TriSb92 was defined by cryo-EM, which revealed triggering of a conformational shift in the Spike trimer rather than competition for ACE2 binding as the molecular basis of its strong inhibitory action. Our results highlight the potential of intranasal inhibitors in protecting susceptible individuals from SARS-CoV-2 infection, and describe a novel type of inhibitor that could be of use in addressing the challenge posed by the Omicron variant.

\section{INTRODUCTION}

The success of the current vaccines in the fight against the SARS-CoV-2 pandemic is challenged by the emergence of novel viral variants of concern (VOCs) including Omicron that show resistance to neutralizing antibodies, the faster than expected waning of vaccine-induced protective immunity, and the prevalent vaccine hesitancy in the population. Moreover, immune disorders or other health conditions can preclude appropriate vaccine responses in many individuals. In addition to pharmaceuticals for treating COVID-19 disease, new approaches for preventing transmission and spreading of SARS-CoV-2 are therefore urgently needed.

Since the nasal epithelium of the respiratory tract is the first dominant replication site of SARS-CoV-2 preceding virus transport into the lung ${ }^{1}$, intranasal administration of SARS-CoV-2 neutralizing agents poses an attractive prophylactic concept that could profoundly impact the course 
and severity of the disease. In animal models, monoclonal antibodies targeted against the Spike envelope protein of SARS-CoV-2 have shown to be effective for COVID-19 prophylaxis ${ }^{6,7}$. To avoid mutational escape from neutralization, such antibodies have been used as cocktails and targeted against conserved regions of Spike ${ }^{8}$. Markedly increased potency could also be achieved by constructing a pentameric IgM version of Spike-targeted IgG antibodies ${ }^{4}$.

To develop more economical and manufacturable anti-SARS-CoV-2 reagents, many laboratories have moved from monoclonal antibodies to smaller antibody fragments, including single variable domain-based nanobodies. Several of these have shown promising antiviral efficacy in cell culture or animal models, including utility as an intranasal SARS-CoV-2 prophylaxis ${ }^{9-12}$.

Compared to these, small antibody-mimetic scaffold proteins are even more robust, versatile to engineer, and cheaper to produce for diverse biological targeting purposes ${ }^{13}$. Sherpabodies (SH3 Recombinant Protein Affinity) are very small ( $<60$ aa) targeting modules that constitute a novel addition to the toolbox of scaffold targeting proteins and comprise of an improved SH3-domain fold derived from the human ciliary adaptor protein nephrocystin ${ }^{14}$. Here, we report engineering of a Sherpabody targeted against a highly conserved region in the Spike receptor binding domain (RBD) into a potent prophylactic anti-SARS-CoV-2 agent.

\section{RESULTS}

\section{Development of Sherpabody-based SARS-CoV-2 inhibitors}

With the aim of developing a novel class of inhibitory proteins that could be used for neutralizing SARS-CoV-2, we screened a large antibody-mimetic phage library displaying $\sim 10^{11}$ unique Sherpabodies (Fig. 1A and B) by using the receptor binding domain (RBD) of the Spike glycoprotein of the Wuhan-Hu-1 strain as an affinity bait. Starting from the second round of affinity selection and phage amplification (panning), a clear enrichment of RBD-binding clones was observed. After three panning cycles, 192 individual Sherpabody-displaying phage clones were isolated and found to represent 15 different sequences. These 15 unique Sherpabodies were tested in phage-ELISA for binding to RBD-mFc, control $\mathrm{mFc}$, or a monoclonal antibody against the E-tag peptide used for monitoring Sherpabody display efficiency (see Fig. 1B). All 15 Sherpabody clones mediated strong and apparently specific RBD binding (Fig. 1C). Clone number 92 (Sb92) was among the strongest RBD binders and was chosen for further development due to its ability to also bind to RBD of SARS-CoV-1 (see later), suggesting a conserved target epitope that would be unlikely to vary between different variants of SARS-CoV-2. Sb92 was produced as a GST-fusion 
protein in bacteria and its RBD-binding affinity was evaluated using a semi-quantitative antigen capture-ELISA (Fig. 1D), which indicated an affinity $\left(\mathrm{K}_{\mathrm{D}}\right)$ of $30 \mathrm{nM}$.

A.

$$
\text { RT-loop }
$$

EEYIAVGDFXXXXXXDLTFKKGEILLVIEX

XXXXXDGWWIAKDAKGNEGLVPRTYLEPYS

n-Src-loop

C.

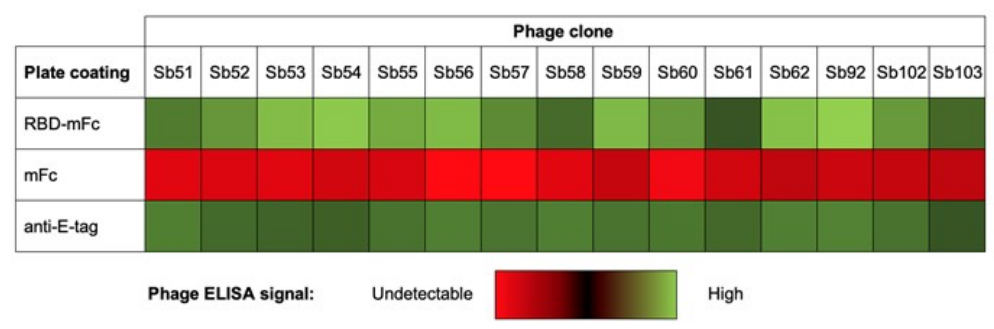

$B$.

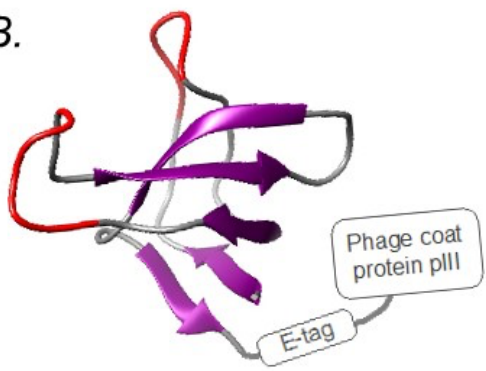

$D$.

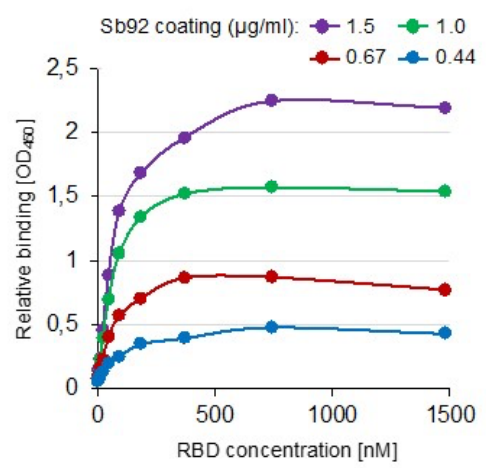

Fig. 1: Discovery of RBD-targeting Sherpabodies. (A-B) Six and three residues in the RT- and n-Srcloops, respectively, of the human nephrocystin SH3 domain were replaced with hexapeptides (in red) comprising random combinations of the 20 natural amino acids to create a large semisynthetic M13 phage library displaying $\sim 10^{11}$ individual Sherpabodies with unique binding surfaces formed by the 12 randomized loop residues shown red in the illustration based on the structure pdb $1 \mathrm{~S}^{1} \mathrm{~N}^{14}$. (C) Heat map showing relative binding in an ELISA assay of fifteen affinity-selected Sherpabody-displaying phage clones targeted to RBD of SARS-CoV-2 Spike protein $(\mathrm{RBD}-\mathrm{mFc})$, negative control $(\mathrm{mFc})$, or an antibody evenly recognizing all displayed clones (anti-E-tag). (D) Semi-quantitative ELISA-based analysis of the RBD-binding affinity ( $30 \mathrm{nM}$ ) of the Sherpabody clone Sb92.

\section{Neutralization of SARS-CoV-2 variants and SARS-CoV-1 by RBD-targeted Sherpabodies}

The potency of Sb92 as a neutralizing agent was tested in a pseudovirus model based on luciferase expressing lentiviral vectors infecting ACE2-expressing HEK293T cells in a Spike-dependent manner ${ }^{15}$. Pseudoviruses carrying Spike proteins corresponding either to the original Wuhan-Hu-1 virus or the B.1.351 (Beta) VOC were incubated with serially diluted concentrations of Sb92 before the infection. Sb92 showed a robust neutralizing capacity with encouraging half-maximal inhibitory 
concentrations $\left(\mathrm{IC}_{50}\right)$ of $7 \mathrm{nM}$ and $28 \mathrm{nM}$ against Beta VOC and Wuhan-Hu-1-based pseudoviruses, respectively (Fig. 2A).

To enhance the potency and utility of Sb92 as a SARS-CoV-2 inhibitor, we constructed a multimerized derivative of Sb92 (TriSb92) containing three tandem copies of Sb92 connected by flexible 15-mer Gly-Ser, (GGGGS)3, linkers. Remarkably, the trimeric architecture of TriSb92 provided it with an almost 200-fold increased potency to neutralize these pseudoviruses, resulting in $\mathrm{IC}_{50}$ values of $150 \mathrm{pM}$ (Wuhan-Hu-1) and $50 \mathrm{pM}$ (Beta VOC) (Fig. 2B). Importantly, also the currently most relevant VOCs Delta and Omicron were potently neutralized by TriSb92 and showed $\mathrm{IC}_{50}$ values of $35 \mathrm{pM}$ and $180 \mathrm{pM}$, respectively (Fig. 2B).
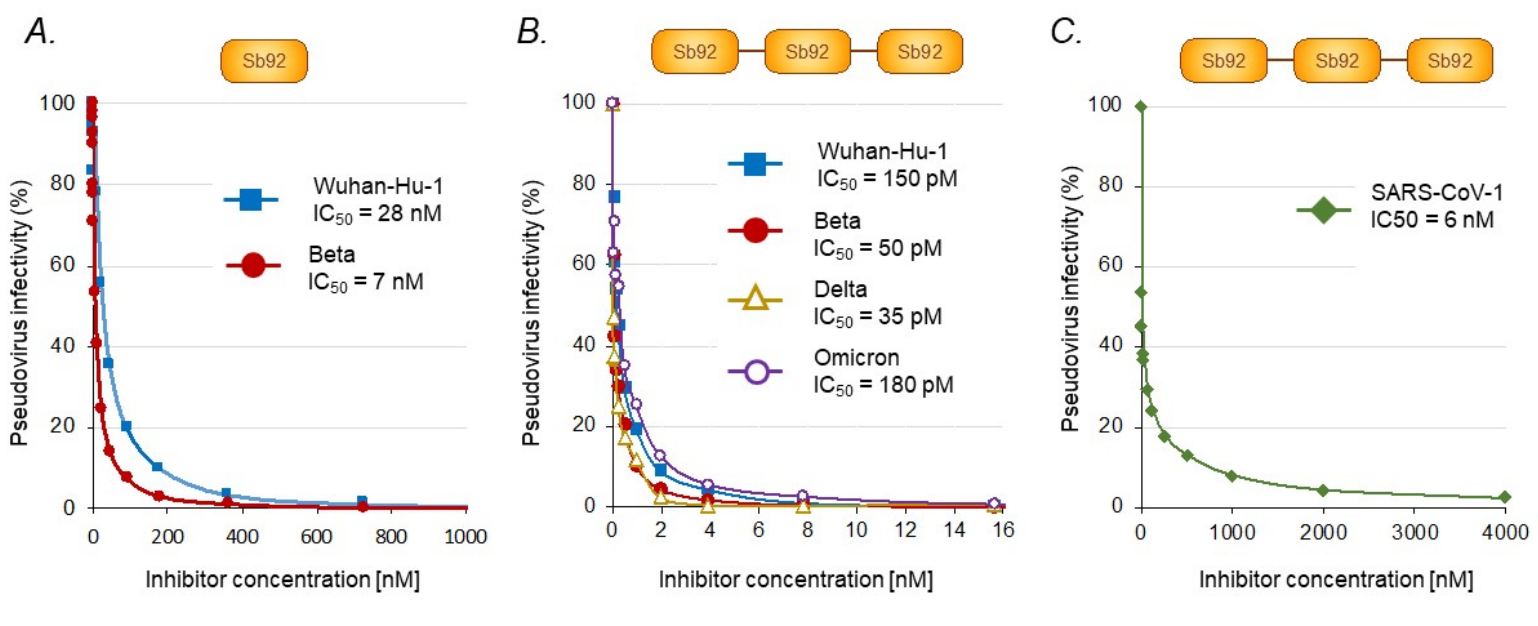

D.

Protective end-point neutralization titers for clinical SARS-CoV-2 variant of concern isolates

$\begin{array}{llll}\text { Alpha (B.1.1.7) } & 1.6 \mathrm{nM} & \text { Kappa (B.1.617.1) } & 2.4 \mathrm{nM} \\ \text { Beta (B.1.351) } & 0.8 \mathrm{nM} & \text { Delta (B.1.617.2) } & 1.2 \mathrm{nM} \\ \mathrm{Mu}(\text { B.1.621) } & 0.6 \mathrm{nM} & \text { Omicron (B1.1.529) } & 6.3 \mathrm{nM}\end{array}$

Fig. 2: Neutralization of SARS-CoV-2 variants and SARS-CoV-1. (A and B) Luciferase expressing pseudoviruses decorated with Spike glycoproteins of the original Wuhan-Hu-1 strains or the Beta, Delta, or Omicron variants of SARS-CoV-2 (C) or SARS-CoV-1 were treated with serially diluted Sb92 (A) or TriSb92 (B and C) before infection of ACE2-expressing target cells. Luciferase activity in these cells is shown relative to normalized to untreated cells set to $100 \%$, and the calculated $\mathrm{IC}_{50}$ concentrations for pseudovirus neutralization are indicated. (D) The potency of TriSb92 in neutralization of clinical isolates of SARS-CoV-2 Alpha, Beta, Delta, Kappa, Mu and Omicron variants was examined using an end-point dilution MNT assay to establish the minimal fully protective TriSb92 concentrations. 
Of note, a pseudovirus carrying the Spike glycoprotein of SARS-CoV-1 could also be readily neutralized by TriSb92, albeit with a somewhat lower efficiency $\left(\mathrm{IC}_{50} 6 \mathrm{nM}\right)$, resembling inhibition of SARS-CoV-2 Spike-mediated infection by the monomeric Sb92 protein (Fig. 2C).

Subsequently, TriSb92 was tested in a classic coronavirus microneutralization test ${ }^{16}$ in VeroE6 cells using clinical SARS-CoV-2 variant isolates. Instead of a half-maximal inhibitory concentration, this robust end-point titration assay reports the last inhibitor dilution that no longer fully protects all replicate culture wells from the cytopathic effect of SARS-CoV-2. Confirming our data from the pseudovirus model, a potent and dose-dependent inhibition was observed for all the SARS-CoV-2 strains tested (Fig. 2D). A complete neutralization could be achieved with very low or subnanomolar concentrations for all the clinical isolates tested, the end-point dilution values being 1.6 nM for Alpha (B.1.1.7), $0.8 \mathrm{nM}$ for Beta (B.1.351), 1.2 nM for Delta (B.1.617.2), $0.6 \mathrm{nM}$ for $\mathrm{Mu}$ (B.1.6219), $2.4 \mathrm{nM}$ for Kappa, (B.1.617.1), and $6.3 \mathrm{nM}$ for Omicron (B1.1.529).

Together, these data show that TriSb92 is an extremely potent inhibitor of SARS-CoV-2 infection, which targets a conserved site in the RBD of Spike in a manner that is insensitive to immune escape mutations found in the relevant VOCs, including Omicron, and provides TriSb92 with the capacity to neutralize a related sarbecovirus (SARS-CoV-1).

\section{Intranasally administered TriSb92 efficiently protects mice against a later challenge with SARS-CoV-2}

To evaluate the prophylactic efficiency of TriSb92 in vivo, we used a recently described animal model of COVID-19 where Balb/c mice are intranasally inoculated with the SARS-CoV-2 Beta (B.1.351) variant. ${ }^{17}$ At different time points before the SARS-CoV-2 challenge mice received intranasal administration of of TriSb92. In the first experiment, mice received $25 \mu \mathrm{g}$ of TriSb92 per nostril and were challenged intranasally one hour later with $2 \times 10^{5}$ PFU of SARS-CoV-2 B.1.351. This amount of TriSb92 was chosen to represent a relatively modest dose, which is in the range also referred as ultra-low in the SARS-CoV-2 antibody inhibitor literature ${ }^{2}$.

Animals were euthanized at 2 days post infection (dpi) and the right lungs subjected to quantitative real-time PCR (RT-qPCR) for viral RNA. In untreated control mice, the lungs exhibited abundant SARS-CoV-2 subgenomic E RNA (median Cq value 18.45), whereas none could be detected in the lungs of the mice that had received a prophylactic dose of TriSb92. These data are shown in Fig. 3A where mouse actin mRNA-normalized sgE RNA quantitation data are 
plotted as percentage of the average sgRNA in the five unprotected control mice. The results were confirmed by histology and immunohistology for the detection of SARS-CoV-2 nucleoprotein (NP) in nasal mucosa, airways and left lungs (Fig. 3B). In control animals, viral antigen was present throughout the respiratory tract, in the nasal cavity (respiratory and olfactory epithelial cells), in bronchiolar epithelial cells and in pneumocytes in adjacent alveoli in association with a mild acute bronchointerstitial pneumonia. Viral antigen was also detected within individual macrophages in bronchiolar lymph nodes. In contrast, treated animals were entirely free of viral antigen in nose, lower airways, lungs and bronchiolar lymph nodes and did not exhibit any pathological changes (Fig. 3B).

A.

B.
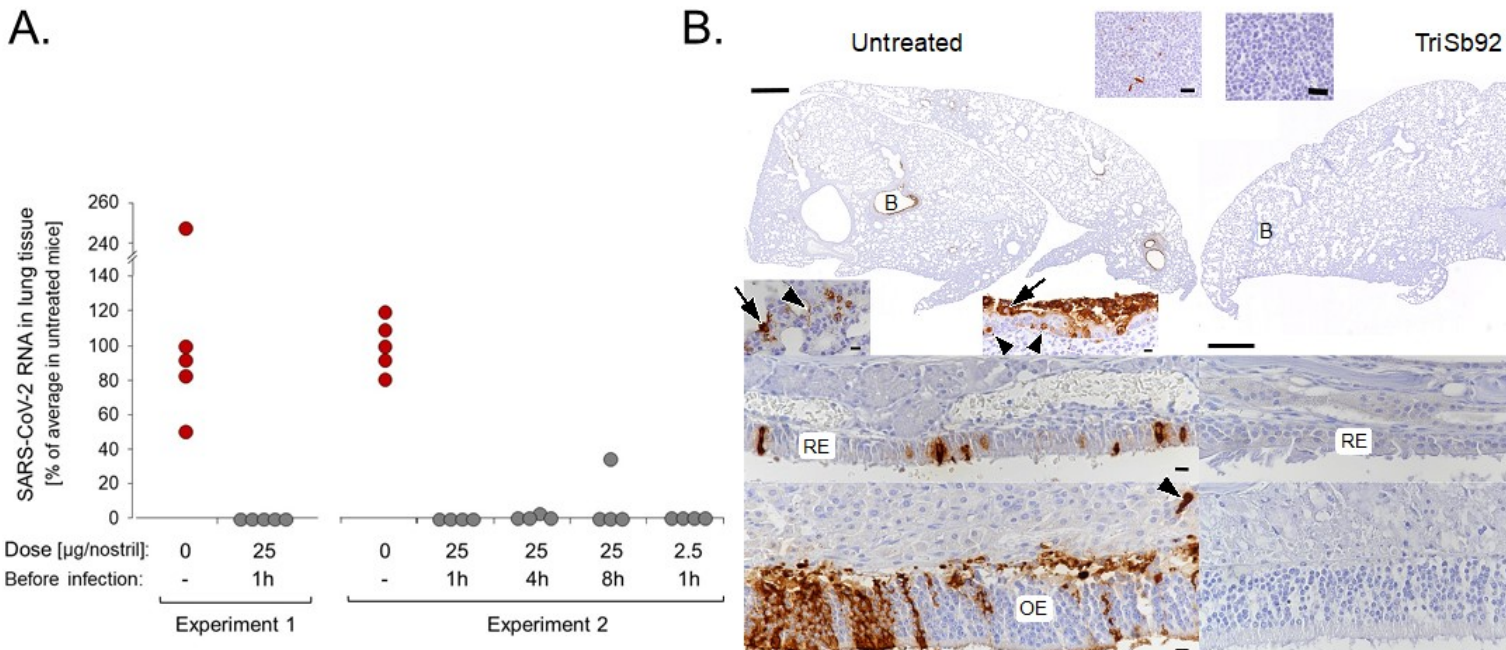
B

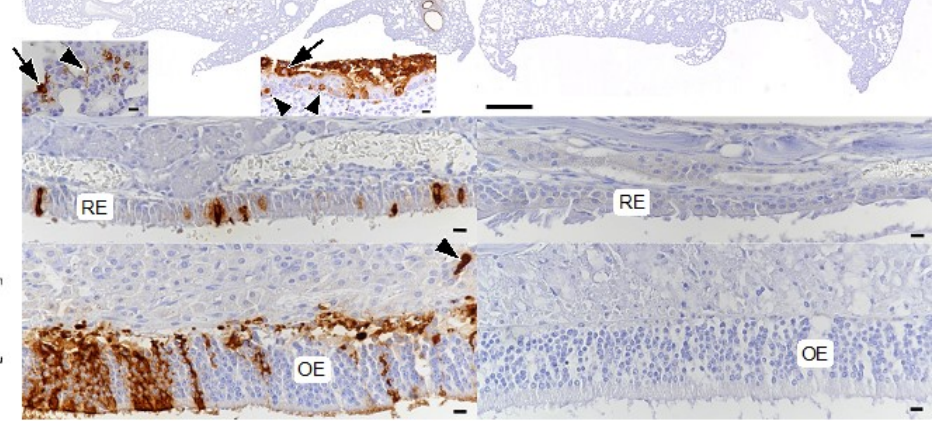

Fig. 3: Prophylactic efficacy of TriSb92 in mice. (A) Subgenomic viral RNA was quantified in lung tissues of Balb/c mice 2 days after challenge with $2 \times 10^{5}$ PFU of SARS-CoV-2 B.1.351 in two independent infection experiments. The infected mice were treated with intranasal administration TriSb92 at the indicated doses and times prior to the infection. (B) SARS-CoV-2 nucleoprotein immunostaining in the nasal cavity and lung tissues 2 days after infection in an untreated mouse (left) and in a mouse pretreated with $25 \mu \mathrm{g}$ of TriSb92 per nostril (right). The untreated animal exhibits viral antigen expression in the nasal respiratory epithelium (RE) and olfactory epithelium (OE). In the lung, several bronchioles (B) exhibit viral antigen in a variable number of epithelial cells that are often degenerate (arrowheads) or sloughed off (arrows). In infected alveoli, both type I pneumocytes (arrowhead) and type II pneumocytes (arrow) are positive. The bronchial lymph node (top inset) exhibits several positive cells (macrophages or dendritic cells). The animal that received TriSb92 does not exhibit any evidence of viral antigen expression in nasal epithelium, lung and bronchial lymph node. Bars: lung overviews $-500 \mu \mathrm{m}$; bronchial lymph nodes $-20 \mu \mathrm{m}$; all others $-10 \mu \mathrm{m}$.

In a second larger experiment, the protective effect of the previously used dose of TriSb92 was compared with a 10 -fold lower dose $(2.5 \mu \mathrm{g}$ per nostril) similarly administered $1 \mathrm{~h}$ before SARS- 
CoV-2 challenge. In addition, we tested the effect of the $25 \mu \mathrm{g}$ per nostril administered $4 \mathrm{~h}$ or $8 \mathrm{~h}$ before virus inoculation. As shown in Fig. 3, also the lower dose of TriSb92 efficiently protected the animals from subsequent SARS-CoV-2 challenge $1 \mathrm{~h}$ later (no detectable sgE RNA in lungs 3 days postinfection); however, in two of these animals viral antigen was detected in a few patches of bronchiolar epithelial cells. Remarkably, TriSb92 was very effective also when given $4 \mathrm{~h}$ before the viral challenge. None of these animals showed evidence of viral antigen expression in the lung, and in only one of the four animals low but detectable levels of sgE RNA were found. Likewise, when TriSb92 was given $8 \mathrm{~h}$ before the challenge the lungs of 3 of the 4 mice were clean of sgE RNA and viral antigen, whereas one animal did show substantial albeit reduced viral RNA levels and also expressed viral antigen within bronchiolar epithelial cells.

Together, these data show that intranasal administration of TriSb92 at relatively low doses can provide an impressive prophylactic protection against SARS-CoV-2 infection in this animal model.

\section{TriSb92 targets a conserved region in the RBD}

To gain structural and mechanistic insight into the broadly cross-neutralizing action of TriSb92, cryogenic electron microscopy (cryoEM) studies were carried out. Single-particle cryoEM analysis of the prefusion SARS-CoV-2 S trimer mixed with TriSb92 resulted in two reconstructions with average estimated resolutions of $3.5 \AA$ and $4.1 \AA$ (Supplementary Fig. 1, Supplementary Table 1) that display the distinct "up" and "down" conformations characteristic of sarbecoviral RBDs ${ }^{18-20}$. The first reconstruction, A, describes the S-trimer with all three RBDs in the up-conformation decorated with additional density corresponding to bound TriSb92 (Fig. 4A and Supplementary Fig. 2). The second reconstruction, B, describes the S-trimer with all three RBDs in the downconformation without any additional density indicative of a Sherpabody (Supplementary Fig. 2). To confirm that the additional density associated with the up-conformation RBDs was TriSb92, we performed a fitting of the SH3 domain of human nephrocystin (PDB 1S1N) ${ }^{14}$ into the additional density, and found that this density perfectly matched with the size and shape of this SH3 backbone of TriSb92 (Supplementary Fig. 3).

As illustrated in Figs. 4B-D, TriSb92 targets a highly conserved region distal from the ACE2 binding site ${ }^{21}$. In line with the observed cross-neutralization (Fig. 2), only a single residue within the predicted binding site of TriSb92 diverges between SARS-CoV-2 and SARS-CoV-1, whereas 
apart from just one of the $15 \mathrm{RBD}$ amino acids changes in Omicron (S375F located at the very edge of the TriSb92 epitope) the mutations found in SARS-CoV-2 VOCs do not involve this binding surface (Fig. 4C-D). The epitope of TriSb92 is distinct from the epitopes of therapeutic monoclonal antibodies (Supplementary Fig. 4) that primarily target the ACE2 binding site and remain vulnerable to amino acid changes accruing in this region. Instead, the epitope of TriSb92 partially overlaps with the 'cryptic epitope' targeted by monoclonal antibody (mAb) CR3022 $2^{21}$ (Supplementary Fig. 4) that does not complete with binding to ACE2. Indeed, the ability of ACE2 and Sb92 to simultaneously bind to the RBD in a non-competitive manner could also be experimentally verified using a sandwich-ELISA approach (Supplementary Fig. 5).

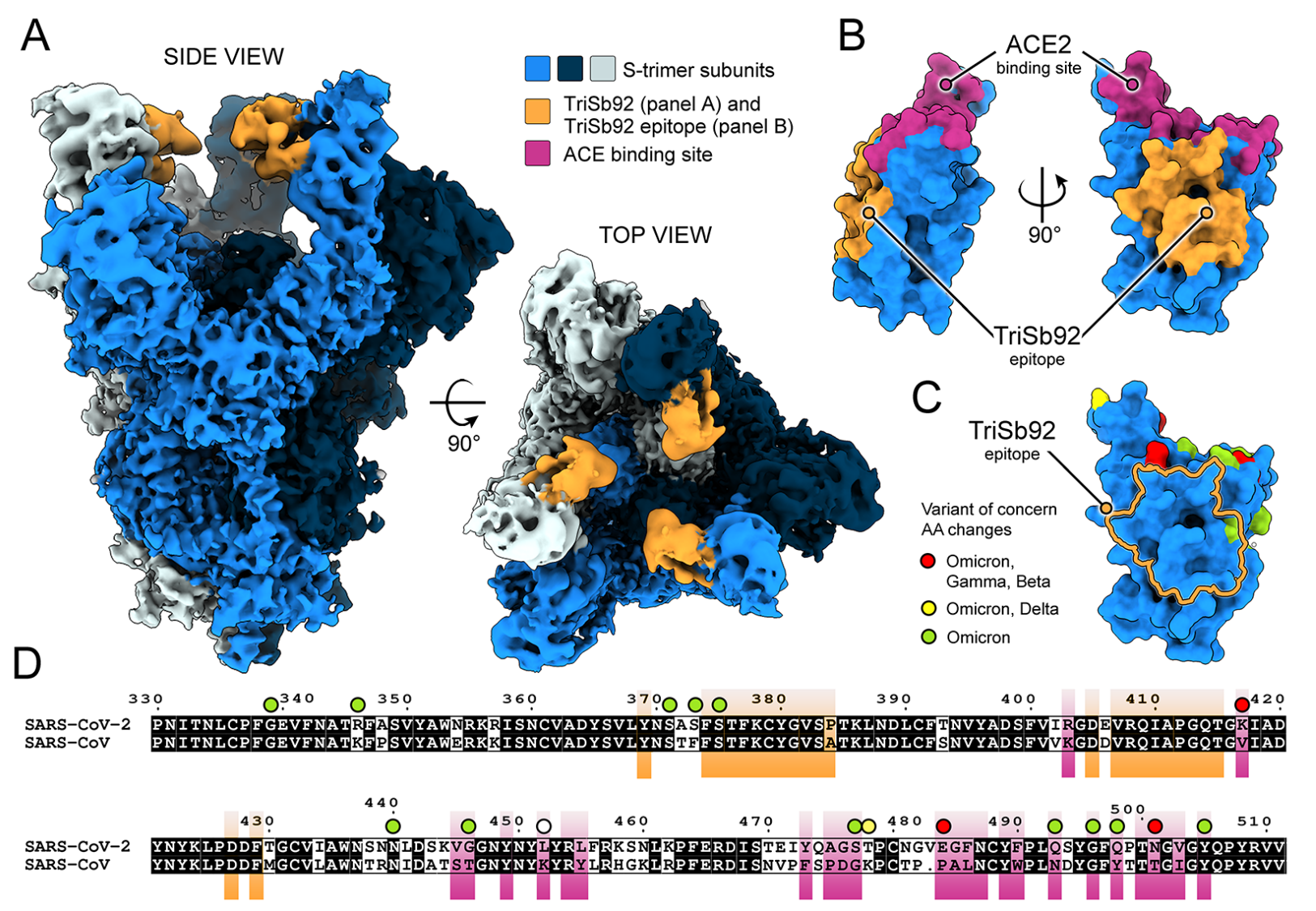

Fig. 4: Cryo-EM of SARS-CoV-2 $\mathrm{S}$ in complex with TriSb92 reveals the highly conserved TriSb92 binding site. (A) Side and top views of the cryoEM reconstruction of Spike trimer in complex with TriSb92. (B) Surface rendering of RBD atomic model with ACE2 binding site and the TriSb92 epitope highlighted (C). Location of the amino acid changes in VOCs relative to the TriSb92 epitope (delineated). (D) Pairwise sequence alignment of SARS-CoV-1 and SARS-CoV-2. The binding sites of TriSb92 and ACE2 are highlighted in orange and in purple, respectively. Circles above the RBD residues indicate amino acid changes in Omicron, Delta, Gamma and/or Beta variants, and are coloured as follows: red is shared by Omicron, Gamma, and Beta; yellow is shared by Omicron and Delta; white is unique to Delta; lime green is unique to Omicron. 


\section{DISCUSSION}

The emergence of new SARS-CoV-2 variants that compromise vaccine efficacy and are resistant to many clinical therapeutic monoclonal antibodies ${ }^{22-27}$ underscores the importance to develop novel molecular tools that potently neutralize VOCs. The urgency of this notion has been strongly emphasized by the recent rise of the potently immune-evasive and fast-spreading Omicron variant. In this study, we describe a promising SARS-CoV-2 inhibitor, TriSb92, based on a new and extremely small antibody-mimetic targeting scaffold termed the Sherpabody. TriSb92 targets a highly conserved region and can neutralize all currently known VOCs, including Omicron, and even a related sarbecovirus SARS-CoV-1. This suggests that TriSb92 could be effective against the future immune escape variants of SARS-CoV-2, and potentially also against emerging novel pandemic sarbecoviruses.

An advantage of Sherpabodies over conventional antibodies and most antibody fragments is that they can be readily assembled into multimers in a context-independent manner to enhance avidity, specificity, as well as functionality. Considering the trimeric nature of the SARS-CoV-2 Spike glycoprotein we leveraged this property of Sherpabodies in the design of TriSb92 to generate a trivalent inhibitor, which increased the neutralization potency of the corresponding RBD-targeted monomeric Sherpabody almost 200 -fold. This provided TriSb92 a capacity to neutralize all VOCs tested with $\mathrm{IC}_{50}$ in the subnanomolar range or very close to it, and to effectively protect mice intranasally treated with as little as 5 ug of TriSb92 from a subsequent challenge with the Beta VOC of SARS-CoV-2 (B.1.351).

Besides the strong avidity effect, the robust SARS-CoV-2 neutralizing potency of TriSb92 appears to stem from its mode of action revealed by our cryoEM studies. Instead of competing with the high-affinity interaction between ACE2 and the RBD of Spike, TriSb92 appears to prevent SARS-CoV-2 entry by triggering an "all three RBDs up" conformation in the Spike trimer. This could destabilize the prefusion spike in a native setting, potentially leading to the spike shedding of the $\mathrm{S} 1$ subunit, as has been demonstrated with certain rare antibodies that also target this region in the $\mathrm{RBD}^{28,29}$. Interestingly, the binding site of TriSb92 is inaccessible in the down-conformation of the RBD. We postulate that once one subunit of TriSb92 can bind to a Spike trimer in a "one RBD up" conformation the remaining two free subunits will promote a conformational shift of the two other RBDs until an all-up conformation is achieved. This model is consistent with our findings, that following TriSb92 treatment the S-trimer presents exclusively in all-up or all-down states.

TriSb92 was built from a Sherpabody clone (Sb92) derived from phage-library screening without any further affinity optimization. Thus, it should be relatively facile to achieve an even 
higher inhibitory potency by affinity maturation of Sb92 and perhaps also by optimization of the trimerization linkers in TriSb92. However, this may not be of great practical relevance considering the already low $\mathrm{IC}_{50}$ concentrations of TriSb92 and the ease of producing it in very high quantities in low-cost bacterial expression systems.

The intranasal dose of TriSb92 that should be administered to susceptible persons prior to events involving a risk for SARS-CoV-2 exposure remains to be established but is likely to be substantially lower than extrapolation of our current data on mice that were challenged by inoculation of the rather massive amount of $2 \times 10^{5}$ PFU of SARS-CoV-2 into their respiratory tract.

Our current data provide a valuable addition to the handful of previous reports on antibodybased approaches for intranasal SARS-CoV-2 prophylaxis ${ }^{2-5}$. A larger body of literature exists on the use of antibodies and other targeted therapies for treatment of COVID-1930,31. While our study focuses on the use of TriSb92 for protection from SARS-CoV-2 infection, these data suggest its use as a therapeutic agent should also be explored. Indeed, the challenge posed by the Omicron variant calls for new innovations both for prevention and treatment of SARS-CoV-2 infection, and TriSb92 could be a valuable addition to the available measures for addressing this situation. 


\section{MATERIALS AND METHDOS}

\section{Phage panning}

Sherpabody phage display library (size $\sim 10^{11} \mathrm{cfu}$ ) was obtained from Next Biomed Therapies Oy. To develop Sherpabodies specific for SARS-CoV-2 spike receptor binding domain (RBD), phage affinity selection process was conducted using standard solid phase sorting strategy. Specific phagedisplayed Sherpabodies were selected for by panning against RBD-mouse IgG2a Fc-fusion protein $^{32}$. Three sequential rounds of off-target depletion and specific panning were performed using a control $\mathrm{mFc}$ and specific RBD-mFc, respectively. The immobilized control and target proteins $(30 \mu \mathrm{g} / \mathrm{ml}$ in PBS; Maxisorp Immunotubes, Nunc) were sequentially incubated in the presence of infectious naïve Sherpabody phage library in $2.5 \%$ milk-PBS- $0.1 \%$ Tween 20 , for $2 \mathrm{~h}$ (RT) and $\mathrm{o} / \mathrm{n}\left(4^{\circ} \mathrm{C}\right)$, respectively. Non-specific phages were removed by extensive washing (PBS$0.05 \%$ PBS-Tween), and the remaining pool of phage were eluted and amplified in E.coli XL1-Blue host cells according to standard protocols. The amplified pool of phages was collected, and the process was reiterated over three rounds to enrich phage-displayed Sherpabodies specific to the RBD-target protein.

\section{Screening and characterization of RBD-binding clones by ELISA}

Representative transformants from the second and third panning rounds were tested for specific binding to the RBD-mFc target protein by enzyme-linked immunosorbent assay (ELISA). Mimicking the affinity selection process, in phage-ELISA the target and control proteins were immobilized on an immunoplate (Maxisorp, Nunc). Each single colony represents progeny from a single E. coli cell that harbours a phagemid expressing a unique Sherpabody-pIII fusion protein. Specifcally, phage-ELISA was performed in 96-well Maxisorp microtiter plates (Nunc) coated over night at $4^{\circ} \mathrm{C}$ with $100 \mu 1$ of target and control proteins (10 $\mu \mathrm{g} / \mathrm{ml}$ in PBS). The wells were washed 3 $\mathrm{x}$ with PBS-0.05\% Tween 20 and blocked with 5\% skimmed milk powder in PBS (milk-PBS) for 2 $\mathrm{h}$ at RT. Appropriate dilutions of Sherpabody-displaying single phage clones were prepared in milk-PBS and incubated with the coated target protein for $1 \mathrm{~h}$ at RT followed by washes $3 \mathrm{x}$ with PBS-0.05\% Tween20 to remove unbound phage. The detection was performed with HRPconjugated mouse monoclonal anti-M13 antibody (GE Healthcare), and TMB (3,3' 5,5'tetramethylbenzidine) substrate. The staining reaction was stopped with $1 \mathrm{M}$ sulfuric acid and absorbance measured at $450 \mathrm{~nm}$ using Hidex Sense Microplate Reader. 
The DNA encapsulated by the positive phage clones was then sequenced and translated to determine the sequence of the displayed Sherpabody.

\section{Sherpabody production and purification}

The discovered unique Sherpabodies were cloned and expressed in E. coli as monomeric and/or trimeric GST-fusion proteins using standard protocols (GE Healthcare). 15 mer Gly-Ser, (GGGGS) 3 , linkers were applied in between each fusion partner. GST-tagged Sherpabodies were purified by affinity chromatography using glutathione sepharose according to manufacturer's instructions (GE Healthcare). The buffer was exchanged to PBS by dialysis (140 $\mathrm{mM} \mathrm{NaCl}, 10 \mathrm{mM}$ phosphate buffer, $3 \mathrm{mM} \mathrm{KCl}, \mathrm{pH} 7.4$ ), and the purified Sherpabodies were stored at $-80^{\circ} \mathrm{C}$ and/or at $+4^{\circ} \mathrm{C}$.

\section{ELISA}

Affinity analysis by semi-quantitative sandwich-ELISA was performed using plastic-coated (o/n, $4^{\circ} \mathrm{C}$ ) GST-Sherpabody fusion protein followed by binding of a concentration series of soluble RBD-His target $\operatorname{protein}^{32}(1 \mathrm{~h}, \mathrm{RT})$. The washes and detection was performed as described above using monoclonal HRP-conjugated anti-His antibody.

GST-Sb92/RBD-His/ACE2-mFc sandwich-ELISAs were performed in two ways: first using plastic-coated GST-Sb92 as the capturing reagent $(10 \mu \mathrm{g} / \mathrm{ml})$ and ACE2-mIgG2a as the detection reagent $(10 \mu \mathrm{g} / \mathrm{ml})$ and then vice-versa $(10 \mu \mathrm{g} / \mathrm{ml}$ and $5 \mu \mathrm{g} / \mathrm{ml}$, respectively) to analyse their ability to simultaneously bind to RBD-His ${ }^{32}$ (2-fold dilution series starting from $10 \mu \mathrm{g} / \mathrm{ml}$ ). The complex formation was detected using either HRP-conjugated goat anti-Mouse-IgG (Sigma Aldrich) or mouse monoclonal anti-GST (GE Healthcare) antibodies. Her2-binding GST-Sb1206 served as a negative control.

\section{Pseudovirus neutralization assay}

HEK293T and HEK293T-ACE2 cells were maintained in DMEM supplemented with 10\% fetal bovine serum, 2\% L-Glutamine, and 1\% penicillin/streptomycin (complete medium). Angiotensinconverting enzyme 2 (ACE2) expressing HEK293T cells were generated by lentivirus-mediated gene transduction. Briefly, pWPI-puro plasmid containing ACE2 cDNA (AB046569.1) was cotransfected with p8.9NdSB and vesicular stomatitis virus G protein (VSV-G) expressing envelope 
plasmids into HEK293T cells in complete medium using polyethylenimine. The recombinant lentivirus containing supernatant was collected $48 \mathrm{~h}$ post-transfection, filtered and used to infect wild-type HEK293T cells. Transduced cells were selected with puromycin.

Luciferase encoding SARS-CoV-2 pseudotyped reporter virus was generated by transfecting HEK293T cells with p8.9NdSB, pWPI-GFP expressing Renilla luciferase, and pCAGGS, an expression vector containing the SARS-CoV-2 S protein cDNA of the Wuhan-Hu-1 reference strain (NC_045512.2). The last 18 amino acids containing an endoplasmic reticulum (ER)-retention signal of the spike protein was removed to enhance transport to the plasma membrane. SARS-CoV-1 pseudovirus was similarly constructed. Pseudovirus stocks were harvested 48 hours after transfection, filtered and stored at $-80^{\circ} \mathrm{C}$. Inserts for the pseudotyping expression vectors producing Spike glycoproteins encoded by the original Wuhan-Hu-1 strain (with D614G mutation) or its variants B.1.351 (Beta), B.1.617.2 (Delta), and B.1.1.529 (Omicron) were assembled from synthetic DNA fragments (Integrated DNA Technologies) using Gibson assembly cloning kit (New England Biolabs) and verified by DNA sequencing.

SARS-CoV-2 pseudovirus-mediated expression of luciferase is directly proportional to the quantity of internalized virus. To assess the inhibition activity, trimeric TriSb92 and monomeric Sb92 and appropriate control proteins were serially diluted in complete medium for desired concentrations. $12.5 \mu \mathrm{l}$ of protein dilutions were mixed with $37.5 \mu 1$ of luciferase encoding SARSCoV-2 pseudotyped reporter viruses in 96-well cell culture plates and incubated at $37^{\circ} \mathrm{C}$ for $30 \mathrm{~min}$. After incubation, 20000 HEK293T-ACE2 cells (in $50 \mu \mathrm{l}$ ) were added on the wells and the plates were further incubated at $37^{\circ} \mathrm{C}$ for $48 \mathrm{~h}$. The amount of internalized pseudovirus in infected cells was quantified by measuring luciferase activity using Renilla-GLO assay (Promega). The relative luciferase units were normalized to those of control samples. Half maximal inhibitory concentrations $\left(\mathrm{IC}_{50}\right)$ were determined from three parallel experiments.

\section{Microneutralization test}

A cytopathic effect (CPE)-based microneutralization (MNT) was performed at the biosafety level 3 laboratory essentially as previously described ${ }^{33}$. Briefly, TriSb92 in duplicates was 2-fold serially diluted in EMEM supplemented with penicillin, streptomycin and $2 \%$ of heat-inactivated fetal bovine serum. Virus was added to obtain 100 x TCID $_{50}$ per well and the 96-well tissue culture plates were incubated for $1 \mathrm{~h}$ at $+37^{\circ} \mathrm{C}, 5 \% \mathrm{CO}_{2}$. African green monkey kidney epithelial (Vero E6) cells were added, following incubation at $+37^{\circ} \mathrm{C}, 5 \% \mathrm{CO}_{2}$ for 4 days. Wells were fixed with $30 \%$ 
formaldehyde and stained with crystal violet. Results were expressed as the lowest TriSb92 concentration that protected the inoculated cultures from CPE caused by productive SARS-CoV-2 infection.

Variant viruses were isolated from patient nasopharyngeal samples. Alpha variant is the isolate hCoV-19/Finland/THL-202102301/2021 (Gisaid ID EPI_ISL_2590786), Beta is hCoV19/Finland/THL-202101018/2021 (EPI_ISL_3471851), Delta is hCoV-19/Finland/THL202117309/2021 (EPI_ISL_2557176), Kappa hCoV-19/Finland/THL-202109869/2021 (EPI_ISL_2506747), $\mathrm{Mu}$ is hCoV-19/Finland/THL-202121391/2021 (EPI_ISL_5934856) and Omicron is hCoV-19/Finland/THL-202126660/2021 (pending for the Gisaid ID). Viruses were propagated (passages 1-2) in VeroE6-TMPRSS2-H10 cells $^{34}$, and further propagated in Vero E6 cells (passage 3) for MNT as described $\mathrm{in}^{35}$.

\section{Animal studies}

Balb/c mice (Envigo) were transported to the University of Helsinki (Finland) biosafety level 3 (BSL-3) facility and acclimatized to individually ventilated biocontainment cages (ISOcage; Scanbur) for seven days with ad libitum water and food (rodent pellets). After the acclimatization period, 9 week old female Balb/c were placed under isoflurane anesthesia and intranasally inoculated with $25 \mu \mathrm{l}$ per nostril of TriSb92 (25 or $2.5 \mu \mathrm{g} /$ nostril). 1, 4 or $8 \mathrm{~h}$ later, the mice were challenged by infection with $20 \mu \mathrm{l}$ of SARS-CoV-2 B.1.351 clinical isolate $\left(2 \times 10^{5}\right.$ PFU). Immediately following the inoculation, the isoflurane was switched off and the animals were held in an upright position for a few seconds to allow the liquid to flush downwards in the nasal cavity. All mice were weighed on a daily basis, and their wellbeing was carefully monitored throughout the experiment for signs of illness (changes in posture or behaviour, rough coat, apathy, ataxia and weight loss), but none of the mice showed any clinical signs. Euthanasia was performed 2 days post infection under terminal isoflurane anesthesia with cervical dislocation. All animals were dissected immediately after death and the right lungs collected for virological examination. The left lung, remaining thoracic organs and heads (animals from the first experiment) were fixed in 10\% buffered formalin for $48 \mathrm{~h}$ and stored in 70\% ethanol for histological and immunohistochemical examinations. Experimental procedures were approved by the Animal Experimental Board of Finland (license number ESAVI/28687/2020). 


\section{RNA isolation and RT-PCR}

RNA was extracted from the right lung of the mice using Trizol (Thermo Scientific) according to the manufacturer's instructions. Isolated RNA was directly subjected to one-step RT-qPCR analysis using TaqMan fast virus 1-step master mix (Thermo Scientific) and AriaMx instrumentation (Agilent) as described previously for E and subE genes ${ }^{36}$. The RT-qPCR for actin was conducted as previously described. ${ }^{37}$ Relative quantification of actin-normalized viral RNA levels was achieved by the comparative $\mathrm{Ct}$ method ${ }^{38}$ using the average of non-treated animals as reference.

\section{Histology and immunohistochemistry}

The left lung, remaining thoracic organs and heads were trimmed for histological examination. Heads were sawn longitudinally in the midline using a diamond saw (Exakt 300; Exakt) and gently decalcified in RDF (Biosystems) for 5 days at room temperature and on a shaker. Tissues were routinely paraffin wax embedded. Consecutive sections $(3-5 \mu \mathrm{m})$ were prepared from lungs and heads and routinely stained with hematoxylin-eosin (HE) or subjected to immunohistochemistry for the detection of SARS-CoV-2 antigen, as previously described ${ }^{17}$.

Recombinant SARS-CoV-2 S trimer production and complex formation. To express the SARSCoV-2 S, a gene encoding for the prefusion stabilized S protein ectodomain ${ }^{19}$ was produced as synthetic cDNA (GeneArt, Life Technologies). The cDNA template encoded for the residues 14-1208 of the original Wuhan-Hu-1 strain S protein (NCBI Reference Sequence: YP_009724390.1) with prefusion-stabilizing proline substitutions at residues 986 and 987, an abrogated furin S1/S2 cleavage site with a "GSAS" substitution at residues 682-685, and a Cterminal T4 fibritin trimerization motif followed by an HRV3C protease cleavage site, SpyTag003, and 8xHisTag. The gene was cloned into the mammalian expression vector pHLsec (Adgene) and transfected into Expi293FTM (Thermo Fisher Scientific) suspension cells at a density of $3 \times 10^{6}$ cells per $\mathrm{ml}$ using the ExpiFectamine ${ }^{\mathrm{TM}} 293$ Transfection Kit (Thermo Fisher Scientific). Following 6 days of cultivation on an orbital shaker a $36.5^{\circ} \mathrm{C}$ and $5 \% \mathrm{CO} 2$, the S protein containing supernatant was collected, clarified by centrifugation, and filtered through a $0.45 \mu \mathrm{M}$ filter. Imidazole was added to the supernatant to $3 \mathrm{mM}$ final concentration, and SARS-CoV-2 S protein was purified from the supernatant by immobilized nickel affinity chromatography with a 1-ml HisTrap excel column (Cytiva) using $300 \mathrm{mM}$ imidazole for elution. S-protein containing eluate was concentrated and buffer exchanged to $10 \mathrm{mM}$ Tris $\mathrm{pH} 8+150 \mathrm{mM} \mathrm{NaCl}$ buffer using an Amicon Ultra 
centrifugal filter (MWCO $100 \mathrm{kDa}$, Millipore). Prior to grid preparation, pure TriSb92 was added to a purified S-trimer aliquot at $1.5 x$ molar excess, and the complex was incubated on ice for 15 minutes.

Cryo-EM grid preparation, data acquisition and data processing. A 3- $\mu$ l aliquot of a pure, prefusion SARS-CoV-2 S-trimer $(0.3 \mathrm{mg} / \mathrm{ml})$ mixed with TriSb92 $(0.05 \mathrm{mg} / \mathrm{ml})$ was applied on Quantifoil 1.2/1.3 grids (1.2- $\mu \mathrm{m}$ hole diameter, 200 mesh copper) that had been glow discharged in a plasma cleaner (PDC-002-CE, Harrick Plasma) for $30 \mathrm{~s}$. The grids were blotted for $8 \mathrm{~s}$ and plunged into liquid ethane using a vitrification apparatus (Vitrobot, Thermo Fisher Scientific). Data were collected on a Titan Krios transmission electron microscope (Thermo Fisher Scientific) equipped with Gatan K2 direct electron detector using electron exposure of $55 \mathrm{e}^{-} / \AA^{2}$ per image at a nominal magnification of $165000 x$, resulting in a pixel size of $0.82 \AA$ (Supplementary Table 1). Data were processed in cryoSPARC ${ }^{39}$. Contrast transfer function parameters were estimated by CTFFIND $4^{40}$. Initial refinements applying no symmetry revealed two conformations, 'all RBDs up' and 'all RBDs down'. These were further separated by hetero refinement applying C3 symmetry. After local motion correction, particles in each conformation were further refined with non-uniform refinement applying C3 symmetry. Resulting maps were filtered to local resolution for further analysis. CryoEM data collection and processing statistics are presented in Supplementary Table 1.

Fitting SARS-CoV-2 S and human nephrocystin SH3 domain structures into the cryo-EM reconstructions. A molecular model of the SARS-CoV-2 S-trimer, derived from PDB 7A2941, was docked into our reconstruction A (Supplementary Figure 1) of the SARS-CoV-2 spike using the 'fitmap' function in UCSF Chimera ${ }^{42}$. In short, a map was simulated for the S-trimer structure to the resolution of $3.5 \AA$, to match the resolution of the spike reconstruction, and fitting was performed using fitmap global search. The top solution yielded a map-to-map correlation score of 0.74, and an exact match between structure and density in all other regions except for the RBDs. Unlike structure 7A29, which presents a "2-up, 1-down” RBD conformation, our reconstruction of the S-trimer displays a "3-up" conformation. In order to account for this difference, an RBD was separated from the spike structure in $\operatorname{Coot}^{43}$ and fitted into the three RBD densities present on the Strimer reconstruction, taking into account the $\mathrm{C} 3$ symmetry of the spike. RBD placement yielded a correlation score of 0.8, completing the fitted S-trimer model (Supplementary Fig. 2). Furthermore, additional density not comprised of the S-protein was identified adjacent to each RBD (Figure 4). To confirm whether this density was comprised of Sb92, the cryoEM reconstruction was segmented 
around the SARS-CoV-2 S-protein using 'color zone' (coloring radius of $3 \AA$ ) and 'split map' functions in Chimera, followed by fitting the structure of human nephrocystin SH3 domain (PDB $1 \mathrm{~S} 1 \mathrm{~N}$ ) into the additional density with a fitmap global search. The top solution placed the SH3 domain into the density adjacent to RBDs with a correlation score of 0.88 . Furthermore, the top solution places SH3 loops RT and n-Src, that contain antigen-binging mutations in Sb92 (Figure 1), on the RBD interface (Supplementary Figure 3), and points the termini of the protein towards the other Sb92 modules, which is line with the expected binding mode of TriSb92.

Our second reconstruction B (Supplementary Figure 1), of a closed SARS-CoV-2 spike, was fitted with SARS-CoV-2 structure (PDB 6ZP044, presenting a "3-down" RBD conformation). Fitmap global search was performed between a map simulated for structure 6ZP0 at resolution of $4.1 \AA$, and our cryoEM reconstruction $\mathrm{B}$, yielding a map-to-map correlation score of 0.83 and leaving no unexplained density (Supplementary Fig. 2).

Molecular graphics and protein interface analysis. Molecular graphics images were generated using PyMOL (The PyMOL Molecular Graphics System, Version 2.4.0a0, Schrödinger, LLC), UCSF Chimera ${ }^{42}$ and ChimeraX, developed by the Resource for Biocomputing, Visualization, and Informatics at the University of California ${ }^{45}$. Residues comprising the interface of SARS-CoV-2 S with ACE2 receptor and inhibitors, including TriSb92 and nanobodies $\mathrm{VHH}-\mathrm{V}$ and Nb30, and the ACE2 receptor and inhibitors including TriSb92 and therapeutic monoclonal antibodies were identified using the PDB ePISA server.

\section{Data availability}

The cryo-EM density maps of i) SARS-CoV-2 spike with RBDs in the "up"-conformation and decorated with TriSb92 (accession code EMD-25781) and ii) SARS-CoV-2 spike with RBDs in the "down" conformation (EMD-25782) have been deposited in the EMDB at the EBI. Fitted SARSCoV-2 spike coordinates have deposited in the PDB (accession codes PDB 7TAQ and 7TAR, for the "up" and "down" conformations, respectively). All data generated or analysed during this study are included in the main text and its supplementary information files. 


\section{Contributions}

ARM and KS designed the study and analysed the data. ARM, HU, PS, JH, LL, RF, KS were responsible for the inhibitor development, characterization and validation. AJ, RN and OR produced and purified the recombinant proteins. AH and PÖ performed the studies with the clinical virus isolates. RK, LK, TSt, TSi, OV were involved in the infectious mouse model and animal studies. AK conducted mouse pathology and immunohistochemistry. Structural biology studies were designed, conducted and analyzed by IR, LH and JTH.

\section{Acknowledgements}

We thank Virpi Syvälahti and Sanna Mäki for expert technical assistance, Mikael Ritvos for help with recombinant protein production, and Michael Hall, Benita Löflund, and Pasi Laurinmäki for support with electron microscopy. We are grateful for the facilities and expertise provided to us by the HiLIFE CryoEM unit and the FIMM Genomic Center at the University of Helsinki, the Umeå Core Facility for Electron Microscopy, CSC - IT Center for Science, Finland, and the Histology Laboratory of Institute of Veterinary Pathology at the University of Zurich. This work was supported by the Academy of Finland (grants 336492 to JH, 342988 to IR, 336490 and 339510 to OV, 336425 and 331787 to KS), EU Horizon 2020 programme (grant 874735 to OV) as well as funding from the Jane and Aatos Erkko Foundation (OV) and the Sakari Alhopuro Foundation $(\mathrm{KS})$. 


\section{References}

1. Hou, Y.J. et al. SARS-CoV-2 reverse genetics reveals a variable infection gradient in the respiratory tract. Cell 182, 429-446 e414 (2020).

2. Nambulli, S. et al. Inhalable Nanobody (PiN-21) prevents and treats SARS-CoV-2 infections in Syrian hamsters at ultra-low doses. Sci Adv 7 (2021).

3. Huo, J. et al. A potent SARS-CoV-2 neutralising nanobody shows therapeutic efficacy in the Syrian golden hamster model of COVID-19. Nat Commun 12, 5469 (2021).

4. $\mathrm{Ku}$, Z. et al. Nasal delivery of an IgM offers broad protection from SARS-CoV-2 variants. Nature 595, 718-723 (2021).

5. $\mathrm{Wu}, \mathrm{X}$. et al. A potent bispecific nanobody protects hACE2 mice against SARS-CoV-2 infection via intranasal administration. Cell Rep 37, 109869 (2021).

6. Baum, A. et al. REGN-COV2 antibodies prevent and treat SARS-CoV-2 infection in rhesus macaques and hamsters. Science 370, 1110-1115 (2020).

7. Hartman, A.L. et al. SARS-CoV-2 infection of African green monkeys results in mild respiratory disease discernible by PET/CT imaging and shedding of infectious virus from both respiratory and gastrointestinal tracts. PLoS Pathog 16, e1008903 (2020).

8. Du, L., Yang, Y. \& Zhang, X. Neutralizing antibodies for the prevention and treatment of COVID-19. Cell Mol Immunol 18, 2293-2306 (2021).

9. Wrapp, D. et al. Structural basis for potent neutralization of betacoronaviruses by single-domain camelid antibodies. Cell 181, 1004-1015 e1015 (2020).

10. Schoof, M. et al. An ultrapotent synthetic nanobody neutralizes SARS-CoV-2 by stabilizing inactive Spike. Science 370, 1473-1479 (2020).

11. Xiang, Y. et al. Versatile and multivalent nanobodies efficiently neutralize SARS-CoV-2. Science 370, 1479-1484 (2020).

12. $\mathrm{Xu}$, J. et al. Nanobodies from camelid mice and llamas neutralize SARS-CoV-2 variants. Nature 595, 278-282 (2021).

13. Simeon, R. \& Chen, Z. In vitro-engineered non-antibody protein therapeutics. Protein Cell 9, 3-14 (2018).

14. le Maire, A. et al. Solution NMR structure of the SH3 domain of human nephrocystin and analysis of a mutation-causing juvenile nephronophthisis. Proteins 59, 347-355 (2005).

15. Crawford, K.H.D. et al. Protocol and reagents for pseudotyping lentiviral particles with SARS-CoV-2 spike protein for neutralization assays. Viruses 12 (2020).

16. Sui, J. et al. Potent neutralization of severe acute respiratory syndrome (SARS) coronavirus by a human $\mathrm{mAb}$ to $\mathrm{S} 1$ protein that blocks receptor association. Proc Natl Acad Sci U S A 101, 2536-2541 (2004). 
17. Kant, R. et al. Common laboratory mice are susceptible to infection with the SARS-CoV2 beta variant. Viruses 13 (2021).

18. Shang, J. et al. Structural basis of receptor recognition by SARS-CoV-2. Nature 581, 221-224 (2020).

19. Walls, A.C. et al. Structure, function, and antigenicity of the SARS-CoV-2 spike glycoprotein. Cell 181, 281-292 e286 (2020).

20. Yuan, Y. et al. Cryo-EM structures of MERS-CoV and SARS-CoV spike glycoproteins reveal the dynamic receptor binding domains. Nat Commun 8, 15092 (2017).

21. Yuan, M. et al. A highly conserved cryptic epitope in the receptor binding domains of SARS-CoV-2 and SARS-CoV. Science 368, 630-633 (2020).

22. Planas, D. et al. Reduced sensitivity of SARS-CoV-2 variant Delta to antibody neutralization. Nature 596, 276-280 (2021).

23. Planas, D. et al. Sensitivity of infectious SARS-CoV-2 B.1.1.7 and B.1.351 variants to neutralizing antibodies. Nat Med 27, 917-924 (2021).

24. Chen, R.E. et al. In vivo monoclonal antibody efficacy against SARS-CoV-2 variant strains. Nature 596, 103-108 (2021).

25. Dong, Y. et al. The way of SARS-CoV-2 vaccine development: success and challenges. Signal Transduct Target Ther 6, 387 (2021).

26. Schmidt, F. et al. Plasma neutralization properties of the SARS-CoV-2 Omicron variant. medRxiv (2021).

27. Tada, T. et al. Partial resistance of SARS-CoV-2 Delta variants to vaccine-elicited antibodies and convalescent sera. iScience 24, 103341 (2021).

28. Li, T. et al. Cross-neutralizing antibodies bind a SARS-CoV-2 cryptic site and resist circulating variants. Nat Commun 12, 5652 (2021).

29. Huo, J. et al. Neutralization of SARS-CoV-2 by Destruction of the Prefusion Spike. Cell Host Microbe 28, 445-454 e446 (2020).

30. Siemieniuk, R.A. et al. Antibody and cellular therapies for treatment of covid-19: a living systematic review and network meta-analysis. BMJ 374, n2231 (2021).

31. Taylor, P.C. et al. Neutralizing monoclonal antibodies for treatment of COVID-19. Nat Rev Immunol 21, 382-393 (2021).

32. Jalkanen, P. et al. A Combination of N and S Antigens With IgA and IgG Measurement Strengthens the Accuracy of SARS-CoV-2 Serodiagnostics. J Infect Dis 224, 218-228 (2021).

33. Haveri, A. et al. Serological and molecular findings during SARS-CoV-2 infection: the first case study in Finland, January to February 2020. Euro Surveill 25 (2020). 
34. Rusanen, J. et al. A generic, scalable, and rapid time-resolved forster resonance energy transfer-based assay for antigen detection-SARS-CoV-2 as a proof of concept. mBio 12 (2021).

35. Haveri, A. et al. Persistence of neutralizing antibodies a year after SARS-CoV-2 infection in humans. Eur J Immunol 51, 3202-3213 (2021).

36. Dagotto, G. et al. Comparison of subgenomic and total RNA in SARS-CoV-2 challenged rhesus macaques. J Virol (2021).

37. Zivcec, M., Safronetz, D., Haddock, E., Feldmann, H. \& Ebihara, H. Validation of assays to monitor immune responses in the Syrian golden hamster (Mesocricetus auratus). $J$ Immunol Methods 368, 24-35 (2011).

38. Schmittgen, T.D. \& Livak, K.J. Analyzing real-time PCR data by the comparative C(T) method. Nat Protoc 3, 1101-1108 (2008).

39. Punjani, A., Rubinstein, J.L., Fleet, D.J. \& Brubaker, M.A. cryoSPARC: algorithms for rapid unsupervised cryo-EM structure determination. Nat Methods 14, 290-296 (2017).

40. Rohou, A. \& Grigorieff, N. CTFFIND4: Fast and accurate defocus estimation from electron micrographs. J Struct Biol 192, 216-221 (2015).

41. Custodio, T.F. et al. Selection, biophysical and structural analysis of synthetic nanobodies that effectively neutralize SARS-CoV-2. Nat Commun 11, 5588 (2020).

42. Pettersen, E.F. et al. UCSF Chimera--a visualization system for exploratory research and analysis. J Comput Chem 25, 1605-1612 (2004).

43. Emsley, P. \& Cowtan, K. Coot: model-building tools for molecular graphics. Acta Crystallogr D Biol Crystallogr 60, 2126-2132 (2004).

44. Xiong, X. et al. A thermostable, closed SARS-CoV-2 spike protein trimer. Nat Struct Mol Biol 27, 934-941 (2020).

45. Pettersen, E.F. et al. UCSF ChimeraX: Structure visualization for researchers, educators, and developers. Protein Sci 30, 70-82 (2021). 


\section{Supplementary data}

A RBDs in the 'up' conformation

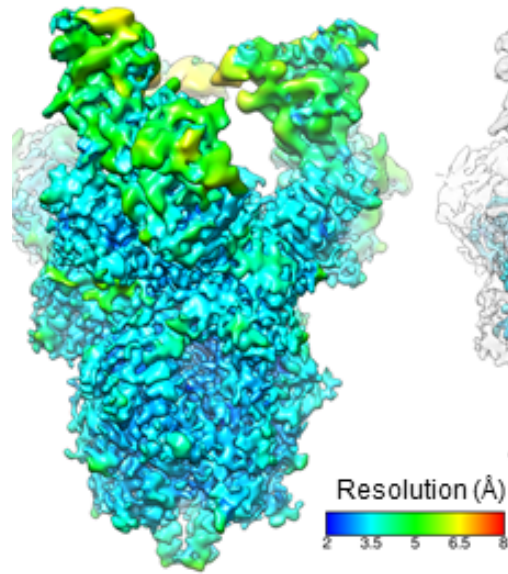

Contour level $\mathbf{0 . 2 6 0}$

C

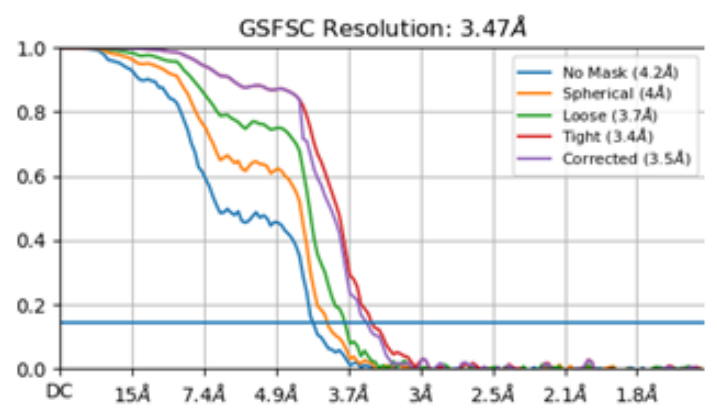

B RBDs in the 'down' conformation

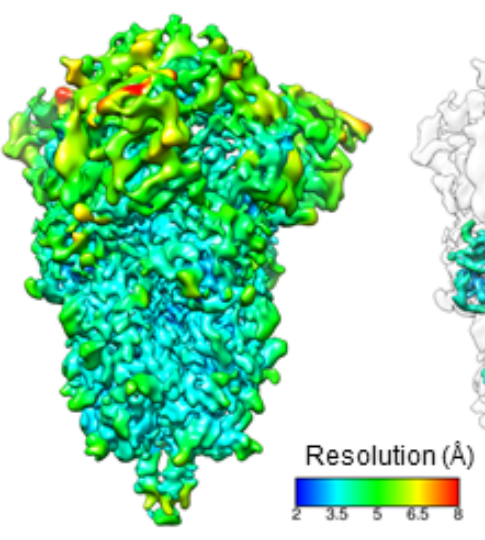

Contour level 0.260

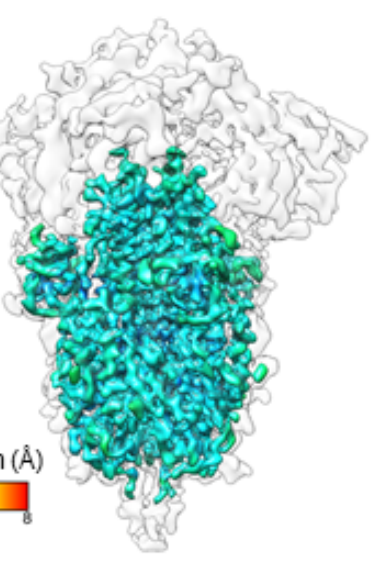

Contour level $\mathbf{0 . 5 3 0}$
$\mathrm{D}$

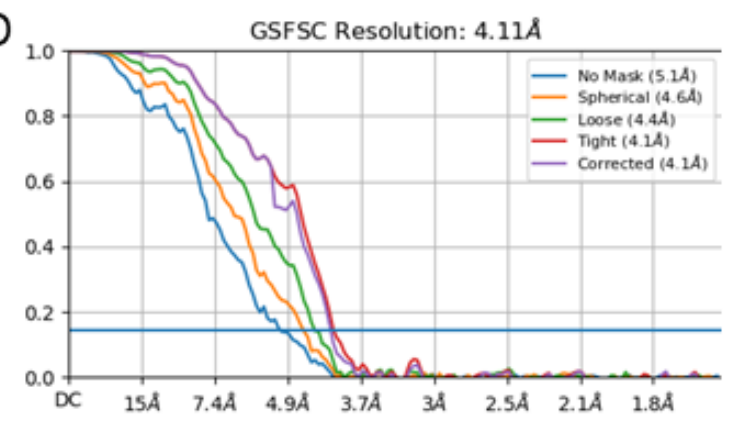

Supplementary Figure 1: CryoEM reconstructions derived from TriSb92-treated SARS-CoV$2 \mathrm{~S}$ trimers. Local resolution was estimated and maps were filtered to local resolution in cryoSPARC'. (A) Reconstruction A describes the S-trimer with all three RBDs in the 'up' conformation, with an average estimated resolution of $3.5 \AA$, while (B) reconstruction $\mathrm{B}$ describes the S-trimer with all three RBDs in the 'down' conformation and has average estimated resolution of $4.1 \AA$. The resolution is highest in the well-ordered S2 subunit, while the intrinsically flexible RBD regions display a more limited 4-5 $\AA$ resolution. (C-D) Panels $C$ and D show the FSC curves for reconstructions $\mathrm{A}$ and $\mathrm{B}$, respectively. 


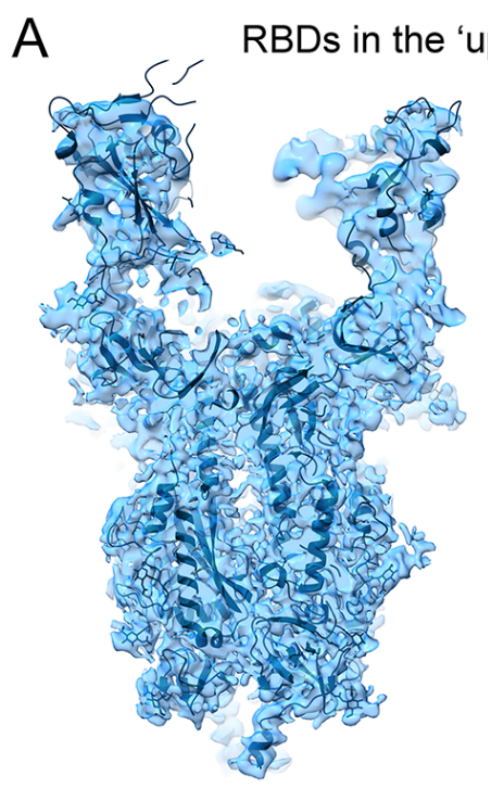

Contour level 0.26

B

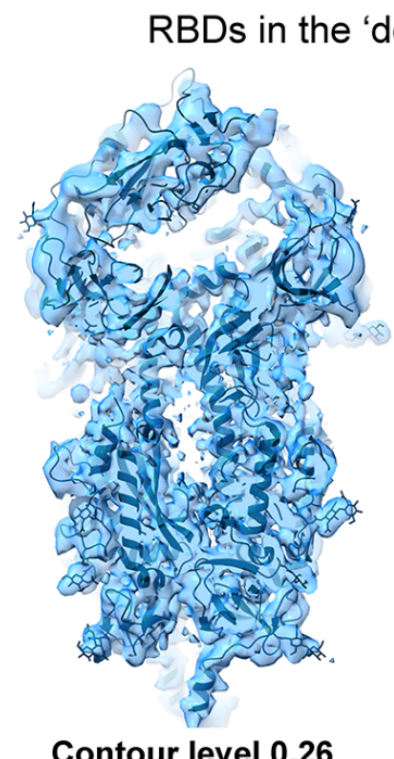

Contour level 0.26

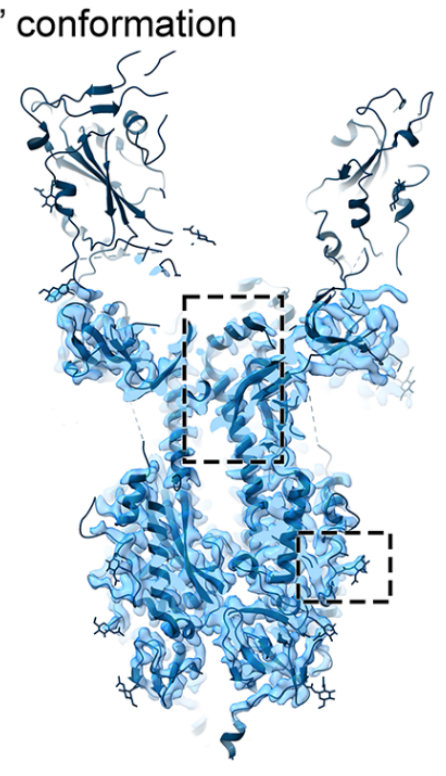

Contour level 0.6
Zoom-in

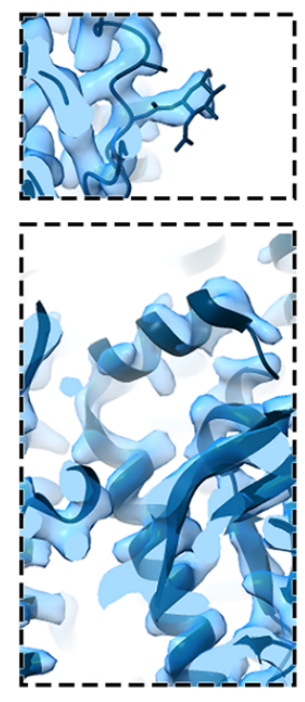

Zoom-in

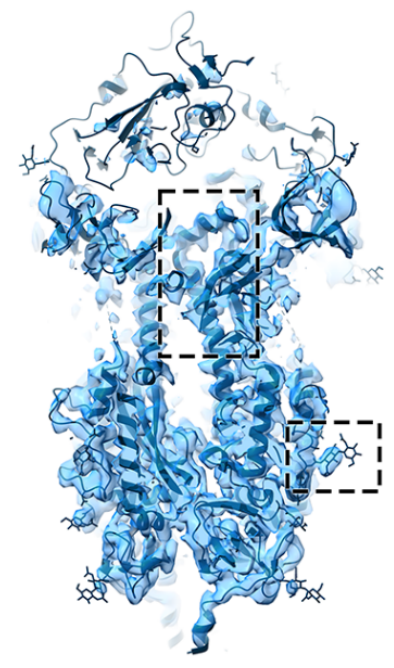

Contour level 0.4

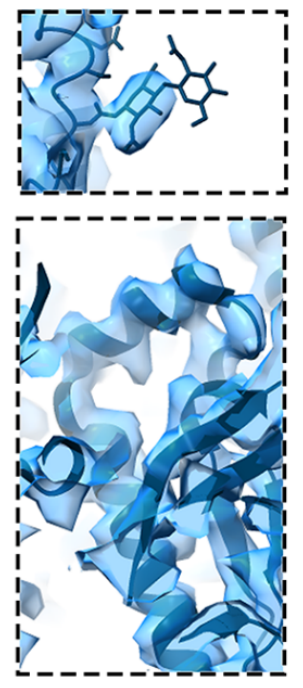

$$
1
$$

Supplementary Figure 2: SARS-CoV-2 S-trimer structures fitted into our cryoEM reconstructions. (A) Cross-section of the cryo-EM reconstruction A with SARS-CoV-2 S structure from PDB 7A292 fitted in as described in the Methods section. (B) CryoEM-reconstruction B of the spike with SARS-CoV-2 structure from PDB $6 \mathrm{ZP0} 0^{3}$ fitted in. Zoom-in panels illustrate the goodness of the fit. 

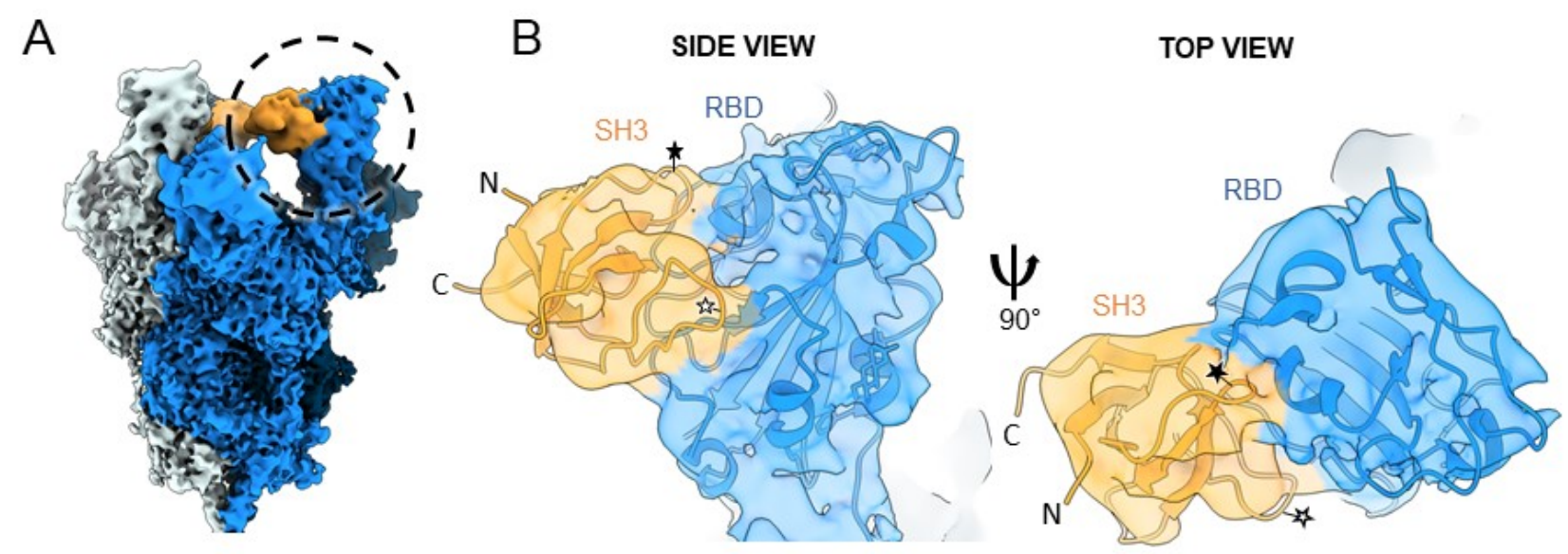

Supplementary Figure 3: Additional density observed in our cryoEM reconstruction matches with the structure of human nephrocystin SH3 domain. (A) Cryo-EM reconstruction of the Spike-TriSb92 complex, with density assigned to $\mathrm{S}$ trimer subunits shown in dark blue, light blue, and pale blue, and the density assigned to TriSb92 in orange. (B) Side and top views of the RBD density region with an RBD molecular model (blue, PDB 7A29) and an SH3 domain model (orange, PDB $1 \mathrm{~S} 1 \mathrm{~N}^{4}$ ) fitted into the density. The RT- and n-Src loop regions of the $\mathrm{SH} 3$ fold that account for target-binding specificity in Sherpabodies (see Fig. 1) are indicated with a white and a black star, respectively. 
A Sb92 and ACE2

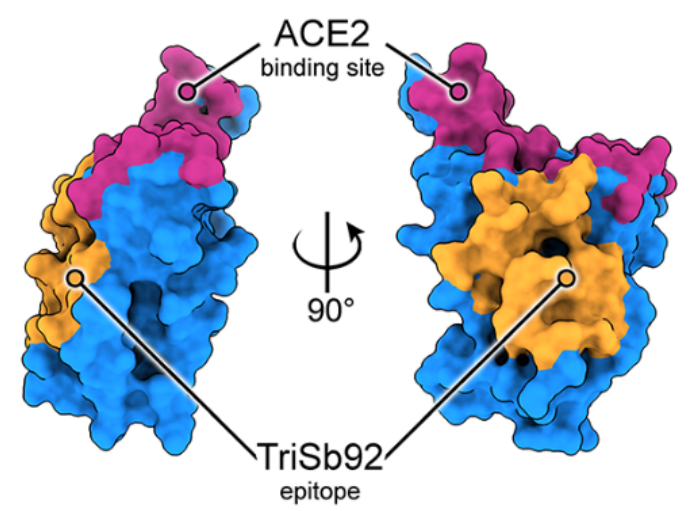

C Bamlanivimab (PDB 7KMG)
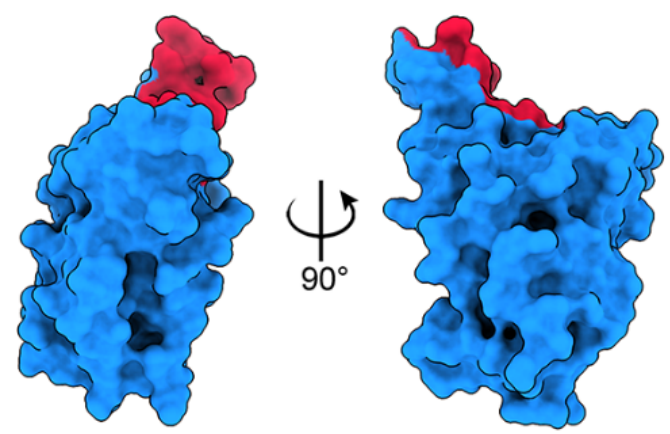

E Casirivimab (PDB 6XDG)
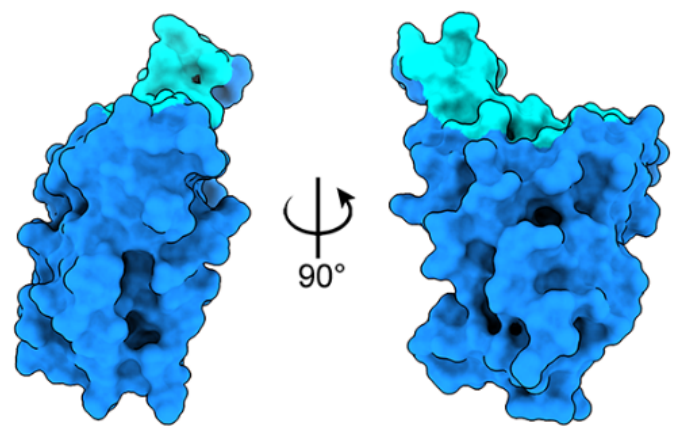

B mAb CR3022 (PDB 6W41)
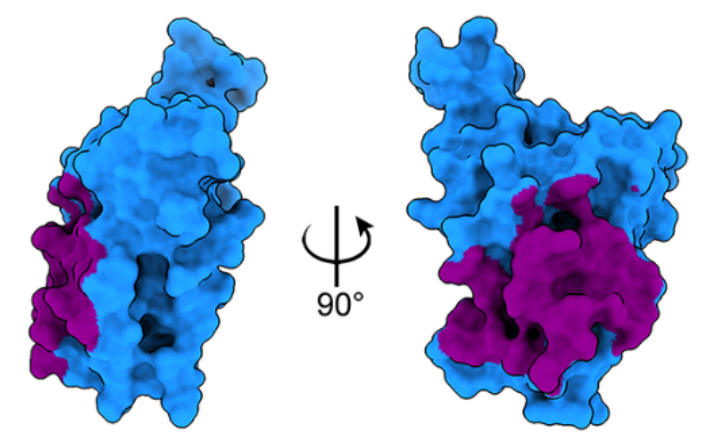

D Etesevimab (PDB 7C01)
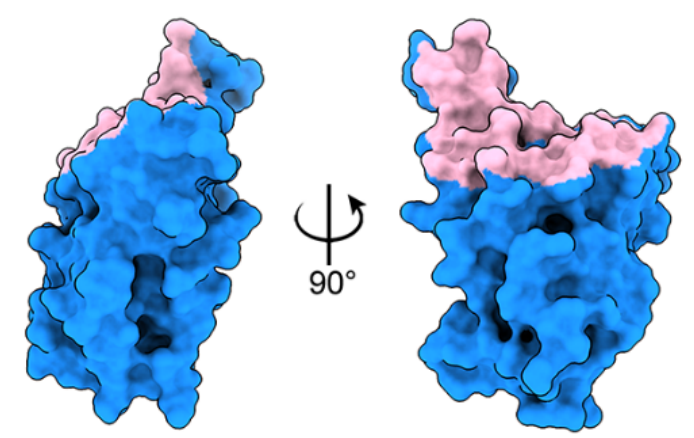

$\mathrm{F}$
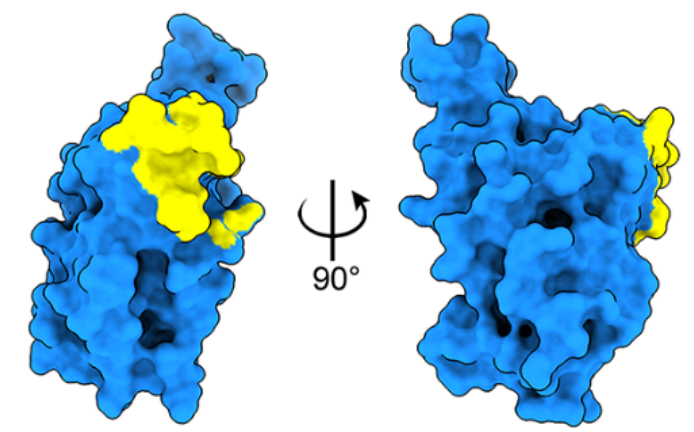

Supplementary Figure 4: Mapping Sb92 epitope in comparison to the epitopes of neutralizing mAbs. A) Sb92 targets an epitope outside of the ACE2-binding region. B) CR3022 (PDB 6W41) ${ }^{5}$ a SARS-CoV-1 neutralizer capable of binding the SARS-CoV-2 RBD, utilizes a conserved and cryptic epitope that overlaps with the Sb92 epitope. C), D), E), F) In contrast to Sb92, therapeutic monoclonal antibodies neutralizing SARS-CoV-2 typically target epitopes that overlap with the ACE2 binding site. These include Bamlanivimab (C, PDB 7KMG) ${ }^{6}$, Etesevimab (D, PDB 7C01) Casirivimab (E, PDB 6XDG) ${ }^{8}$ and Imdevimab (F, PDB 6XDG) ${ }^{8}$. 
A.
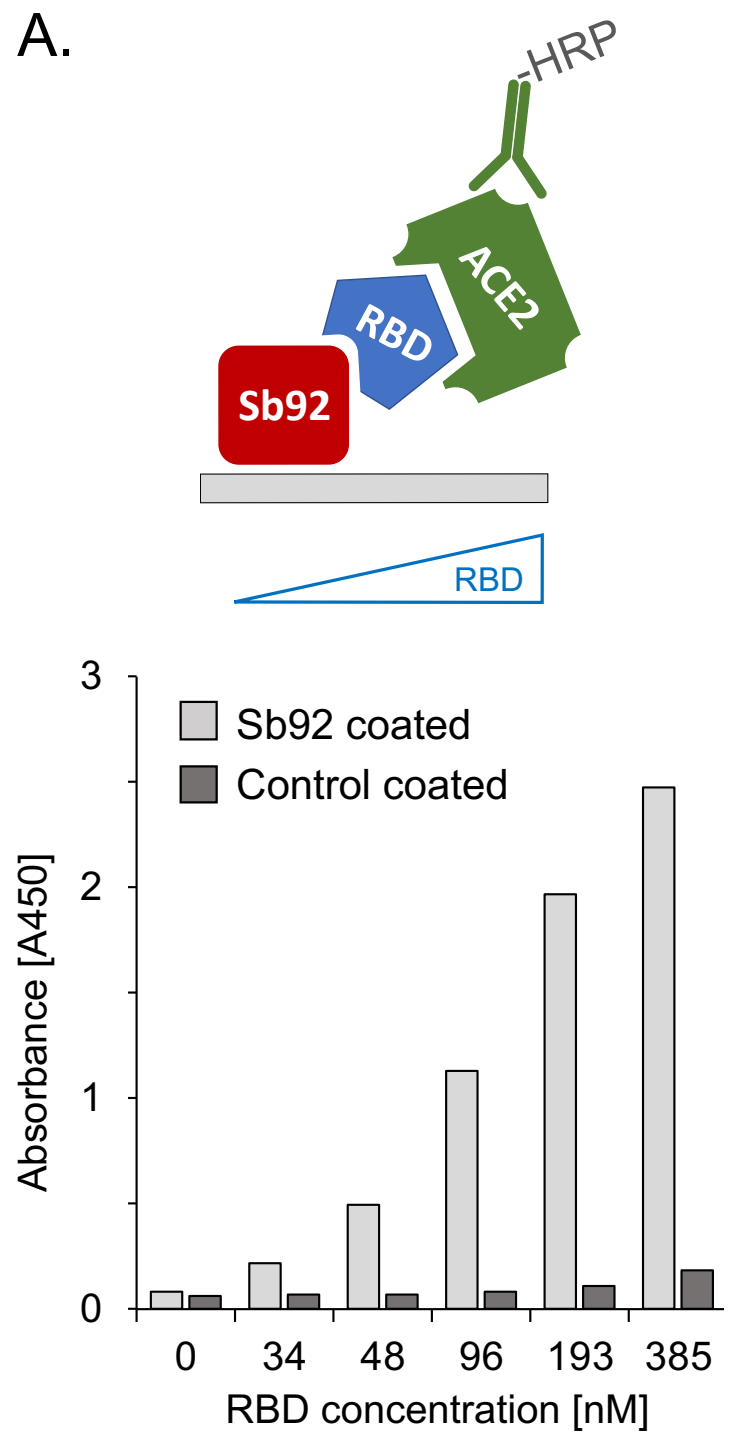

B.
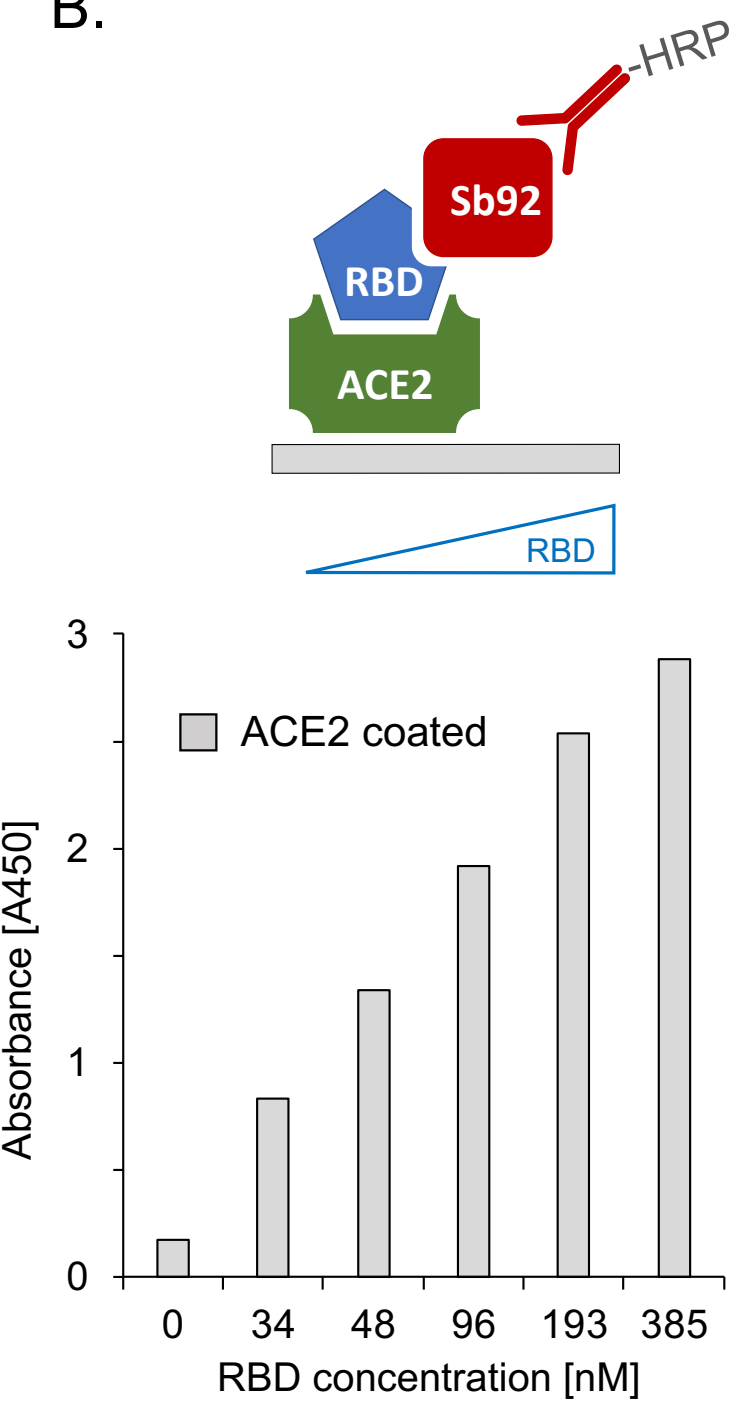

\section{Supplementary Figure 5: Sandwich-ELISAs demonstrating simultaneous binding of Sb92 and}

ACE2 to RBD. ELISAs were performed in two ways: (A) using plastic-coated GST-Sb92 as the capturing reagent and $\mathrm{ACE} 2-\mathrm{mFc}$ as the detector reagent, or (B) vice versa. In (A) a Her2-specific GST-Sb1206 was also tested as a negative control (Control coated). A constant amount of the capturing and detector reagents were used in each case, while increasing concentrations $(0-385$ $\mathrm{nM}$ ) of RBD were added as a bridging reagent to test the formation of a trimeric Sb92-RBD-ACE2 complex, which was visualized using a HRP-conjugated antibody against the detector. 


\section{Supplementary Table1. Cryo-EM data collection and processing statistics.}

\begin{tabular}{|c|c|c|}
\hline & $\begin{array}{l}\text { S-trimer 'all RBDs up' with TriSb92 } \\
\text { EMD-25781 PDB 7TAQ }\end{array}$ & $\begin{array}{l}\text { S-trimer 'all RBDs down' } \\
\text { EMD-25782 PDB 7TAR }\end{array}$ \\
\hline \multicolumn{3}{|c|}{ Data collection and processing } \\
\hline Magnification & $165,000 x$ & $165,000 \mathrm{x}$ \\
\hline Voltage $(\mathrm{kV})$ & 300 & 300 \\
\hline Electron exposure $\left(\mathrm{e}-/ \AA^{2}\right)$ & 55 & 55 \\
\hline Defocus range $(\mu \mathrm{m})$ & $1.5-3.0$ & $1.5-3.0$ \\
\hline Pixel size $(\AA)$ & 0.82 & 0.82 \\
\hline Symmetry imposed & $\mathrm{C} 3$ & $\mathrm{C} 3$ \\
\hline Initial particle images (no.) & 691,665 & 691,665 \\
\hline Final particle images (no.) & 23,530 & 13,847 \\
\hline Map resolution $(\AA)$ & 3.5 & 4.1 \\
\hline FSC threshold & 0.143 & 0.143 \\
\hline Map resolution range $(\AA)$ & $2.3-11.1$ & $2.5-12.0$ \\
\hline
\end{tabular}

\section{Supplementary references}

1. Punjani, A., Rubinstein, J.L., Fleet, D.J. \& Brubaker, M.A. cryoSPARC: algorithms for rapid unsupervised cryo-EM structure determination. Nat Methods 14, 290-296 (2017).

2. Custodio, T.F. et al. Selection, biophysical and structural analysis of synthetic nanobodies that effectively neutralize SARS-CoV-2. Nat Commun 11, 5588 (2020).

3. Xiong, X. et al. A thermostable, closed SARS-CoV-2 spike protein trimer. Nat Struct Mol Biol 27, 934-941 (2020).

4. le Maire, A. et al. Solution NMR structure of the SH3 domain of human nephrocystin and analysis of a mutation-causing juvenile nephronophthisis. Proteins 59, 347-355 (2005).

5. Yuan, M. et al. A highly conserved cryptic epitope in the receptor binding domains of SARS-CoV-2 and SARS-CoV. Science 368, 630-633 (2020).

6. Jones, B.E. et al. The neutralizing antibody, LY-CoV555, protects against SARS-CoV2 infection in nonhuman primates. Sci Transl Med 13 (2021).

7. Shi, R. et al. A human neutralizing antibody targets the receptor-binding site of SARS-CoV-2. Nature 584, 120-124 (2020).

8. Hansen, J. et al. Studies in humanized mice and convalescent humans yield a SARSCoV-2 antibody cocktail. Science 369, 1010-1014 (2020). 